\title{
E-PL: Um Objeto de Aprendizagem para o Ensino de Programação Linear
}

\author{
Rodrigo Veras ${ }^{1}$, Leonardo de Sousa ${ }^{2}$, Marcos Frazão ${ }^{1}$, \\ Iális de Paula $\mathbf{J r}^{3}$, Vinícius Machado ${ }^{1}$ \\ ${ }^{1}$ Departamento de Informática e Estatística (DIE) \\ Universidade Federal do Piauí (UFPI), Teresina - PI, Brasil \\ ${ }^{2}$ Centro de Educação Aberta e a Distância (CEAD) \\ Universidade Federal do Piauí (UFPI), Teresina - PI, Brasil \\ ${ }^{3}$ Curso de Engenharia da Computação - Campus de Sobral \\ Universidade Federal do Ceará (UFC), Sobral - CE, Brasil
}

\{rveras, leonardoramon, marcosfrazao, vinicius\}@ufpi.br, ialis@ufc.br

\begin{abstract}
This paper describes the development of a learning object for the study of the simplex method called E-PL (Teaching Linear Programming). This method is the main topic of Linear Programming (LP) subject which is present in the curricula of several graduated courses, such as those related to Computer Science, Engineering, Accounting and Administration. The LP is an area of research with applications in diverse fields of the scientific, commercial and technical. Nonetheless, in spite of being a very practical discipline, most initiatives in LP teaching/learning adopts a strategy predominantly theoretical. E-PL can be used in classroom or distance teaching because there is a large interactivity with the student and their choices are guided and corrected automatically.
\end{abstract}

Resumo. Este artigo descreve o desenvolvimento de um objeto de aprendizagem para o estudo do método Simplex denominado E-PL (Ensino de Programação Linear). Este método é o principal conteúdo da disciplina de Programação Linear (PL) que está presente em vários cursos superiores ligados à Computação, Engenharia, Contabilidade e Administração. A PL é uma área de pesquisa com aplicações em diversos ramos da iniciativa científica, comercial e técnica. Apesar de seu cunho prático, grande parte das iniciativas de ensino/aprendizagem adota uma estratégia predominantemente teórica. O E-PL pode ser utilizado tanto no ensino presencial quanto a distância devido à interatividade com o aluno e suas escolhas são corrigidas e orientadas automaticamente.

\section{Introdução}

A Pesquisa Operacional (PO) é considerada uma ciência aplicada cujo objetivo é a melhoria da performance em organizações cujo trabalho se dá através da formulação de modelos matemáticos a serem resolvidos com o auxílio de computadores. Esses modelos, dependendo de sua natureza, podem ser solucionados por técnicas matemáticas específicas. Algumas destas técnicas são: Programação Linear, Programação Dinâmica, Programação Inteira, Teoria das Filas, Teoria dos Jogos, Teoria dos Grafos, Planejamento com PERT/CPM, Análise de Risco, Métodos Heurísticos etc [Goldbarg e Luna 2005]. 
Dentre estas, o estudo das técnicas de Programação Linear (PL) é uma área fundamental nos campos da Ciência da Computação, Sistemas de Informação, Engenharia de Produção, Ciências Contábeis, Administração e Estatística, entre outros cursos [Colin 2007]. Em alguns países, o ensino de PL é conteúdo programático das disciplinas de matemática nas escolas [de Almeida Paiva 2008].

Os principais objetivos do ensino de PL são a modelagem, solução e análise de problemas de decisão. A solução destes problemas, chamados de Problemas de Programação Linear (PPL), é feita utilizando-se o método Simplex que foi desenvolvido em 1947 por George B. Dantzig. O Simplex é um algoritmo iterativo utilizado para identificar, algebricamente, a solução ótima de um PPL [Puccini e Pizzolato 1987].

O método Simplex envolve, geralmente, um grande volume de cálculos repetitivos, fazendo-se necessário o uso intensivo do computador. A literatura voltada para o ensino de PL apresenta uma grande variedade de exemplos, porém, sem suporte a recursos computacionais. Assim, é comum o uso de práticas de laboratório e desenvolvimento de projetos como forma de complementação do conteúdo teórico.

Métodos de ensino evoluíram muito ao longo do tempo, desde a forma de abordar os temas de ensino até a forma de apresentá-los a quem está aprendendo. Porém, o maior problema para o provedor da informação é fazer com que esta seja absorvida de forma compreensível, independente do método utilizado.

Com o intuito de produzir melhores resultados no processo de aprendizagem em cursos da área de Computação com um enfoque social [de Souza et al. 2004], faz-se constante a necessidade de apropriação estratégica [Passerino et al. 2006] e atualização das didáticas de ensino de forma geral. Procura-se transformar processos abstratos em concretos através do uso de software, possibilitando melhor compreensão dos tópicos abordados, além de uma maior interação entre o aluno e o objeto de trabalho [Santos et al. 2008].

Portanto, este processo é classificado como software Educativo [Vieira 2003], mais particularmente Objeto de Aprendizagem, por se tratar de uma modelagem de um sistema computacional e de uma ferramenta de Tecnologia da Informação e Comunicação (TIC), auxiliando a educação na relação do ensino com a aprendizagem [Amen e Nunes 2006, da Silva Brito e Menta 2007]. Além disso, permite que educadores e pesquisadores utilizem novos métodos e paradigmas, incluindo de Ensino a Distância para interatividade e interação [da Silva Moro et al. 2008, Bassani 2009].

Este artigo apresenta o E-PL (Ensino de Programação Linear), uma ferramenta de ensino-aprendizagem da disciplina Programação Linear com o objetivo principal de auxiliar na compreensão do método Simplex, estando organizado da seguinte maneira: na próxima seção descrevemos objetos de aprendizagem e sua importância; na seção 3 apresentamos os conceitos de Pesquisa Operacional e Programação Linear; na seção 4 enumeramos os trabalhos relacionados. A seção 5 descreve a ferramenta de ensino desenvolvida e a seção 6 relata o impacto do uso da ferramenta, os benefícios alcançados e os trabalhos futuros.

\section{Objetos de Aprendizagem}

Um Objeto de Aprendizagem (OA) pode ser definido de várias maneiras, mas devese assumir este como um recurso digital ou não digital a ser reutilizado e que ajude 
na aprendizagem. Esses objetos são caracterizados por construir um ambiente virtual para a manipulação e aprendizagem de assuntos específicos. A sua popularidade devese ao fato de ser uma ferramenta de fácil construção e de alta capacidade didática [Schwarzelmüller e Ornellas 2006]. Eles têm uma boa utilização, pois são adequados também para sistemas de ensino a distância.

Objetos de Aprendizagem são armazenados em repositórios, os quais normalmente estão ligados a um sistema de gerenciamento de aprendizagem (Learning Management System - LMS). O LMS, também conhecido como Sistema de Gerenciamento de Cursos (SGC), é um software desenvolvido sobre uma metodologia pedagógica para auxiliar a execução de ensino e aprendizagem virtual ou semipresencial. Estes repositórios são facilitadores na montagem de um planejamento das atividades educacionais nas quais serão utilizados os objetos.

Como exemplos de alguns repositórios de objetos de aprendizagem no Brasil, temos:

- CESTA (http://www.cinted.ufrgs.br/cesta/);

- LabVirt (www.labvirt.fe.usp.br);

- Oe3-tools (http://www.cesec.ufpr.br/etools/oe3);

Outros exemplos, fora do Brasil, são:

- MERLOT (http://www.merlot.org/merlot/);

- CAREO (http://www.ucalgary.ca/commons/careo);

- ARIADNE (http://www.ariadne-eu.org);

- Wisc-Online (http://www.wisc-online.com).

Objetos de aprendizagem para PO e PL são importantes porque relacionam significativamente estudos teóricos de aprendizagem como forma de embasamento ao conteúdo a ser visto futuramente [Marquesi e Silveira 2008], além de atuarem como uma complementação de atividades presenciais.

\section{Pesquisa Operacional e Programação Linear}

Por conta das evasões e dificuldades de aprendizagem ocasionadas por falta de ligações atrativas entre conteúdos e métodos [Pereira et al. 2010], modelos com alternativas de ensino-aprendizagem devem ser criados [Bolgheroni e Silveira 2008, Valente 1993] para tornar extremamente relevante o ensino que desenvolva o engajamento dos alunos em projetos reais na área de Computação. Consequentemente, na disciplina Pesquisa Operacional não poderia ser diferente, em virtude do nível de abstração dos seus temas relevantes.

A PO é uma busca científica das melhores condições de operação de um sistema. Os analistas de PO se dedicam a solução de vários tipos de problemas de operação e organização, como, por exemplo, produção, distribuição e vendas, operações industriais e governamentais. A PO trata da modelagem matemática de fenômenos estáticos ou dinâmicos. Os problemas estáticos são denominados por determinísticos. Nestes problemas, todos os componentes são conhecidos a priori e nenhuma aleatoriedade em sua ocorrência é admitida. Já os problemas dinâmicos são denominados estocásticos e seus elementos apresentam uma probabilidade de ocorrência em uma determinada forma.

Dois eventos motivaram o rápido desenvolvimento da PO. O primeiro foi o desenvolvimento de um algoritmo simples para solucionar problemas de programação linear, 
denominado algoritmo Simplex e proposto por George Dantzig em 1947.O segundo foi a proliferação dos microcomputadores e o rápido aumento em sua velocidade de processamento.

\subsection{Programação Linear}

A Programação Linear visa fundamentalmente encontrar a melhor solução para problemas que tenham seus modelos representados por expressões lineares. A PL consiste na maximização ou minimização de uma função linear denominada função objetivo. Deve-se, entretanto, respeitar um sistema linear de igualdades e/ou desigualdades que recebem o nome de restrições do problema.

As restrições representam recursos disponíveis, tais como capital, mão-de-obra, equipamentos. Ou representam exigências e condições que devem ser cumpridas no problema. Essas restrições do modelo determinam uma região chamada conjunto das soluções viáveis ou região viável. À melhor das soluções viáveis, isto é, aquela que maximiza ou minimiza a função objetivo, denomina-se solução ótima. O objetivo da PL consiste, desta forma, na determinação da solução ótima.

O tipo mais comum de aplicação empregada envolve o problema de distribuir recursos limitados entre atividades que estão competindo entre si da melhor maneira possível. A PL usa um modelo matemático para descrever este tipo de problema. O termo linear significa que todas as funções matemáticas do modelo são, obrigatoriamente, funções lineares. A palavra programação não se refere a programação de computadores, mas sim como um sinônimo de planejamento.

Desta forma, podemos definir a PL como sendo o planejamento de atividades para obter um resultado ótimo. Um resultado que atenda, da melhor forma possível, a um determinado objetivo. Embora a alocação de recursos seja o tipo mais comum, a PL pode ser empregada em várias outras aplicações. Qualquer problema cujo modelo matemático se enquadra na forma geral de um modelo de PL é um PPL. Na literatura, considera-se o método Simplex como extremamente eficiente na solução de PPL's, mesmo com milhares de variáveis.

\subsection{O Método Simplex}

O método Simplex [Puccini e Pizzolato 1987] é uma técnica usada para determinar, algebricamente, a solução ótima de um modelo de programação linear. Havendo uma solução para o modelo, o Simplex a encontra através de um processo de iterações sucessivas do sistema linear modelado, como é sumarizado no Algoritmo 1.

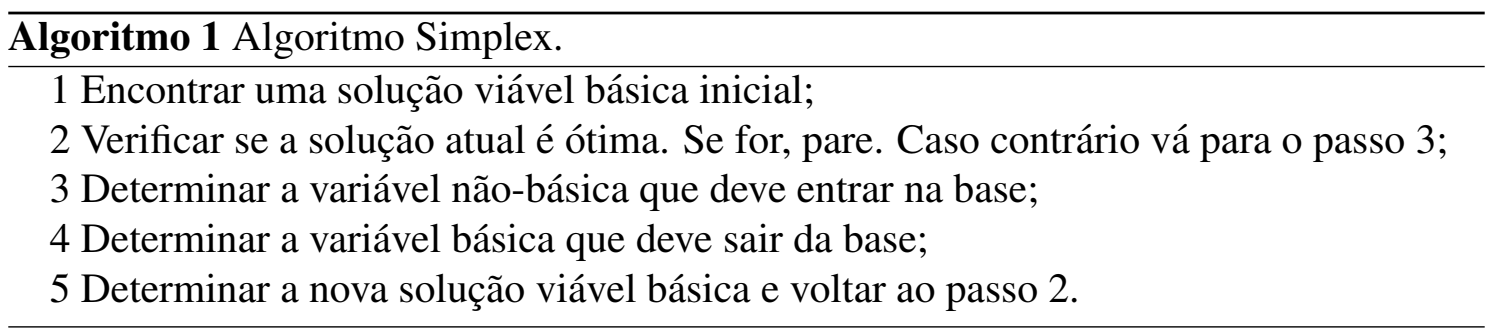

Em resumo, pode-se afirmar que o método Simplex consiste em determinar uma solução inicial viável que será iterativamente melhorada. Essa melhora na solução é feita alterando-se os valores das variáveis do problema (processo de entrada e saída na base). 


\section{Trabalhos Relacionados}

A utilização de técnicas de PO em grande escala fez surgir sistemas que as implementam, sendo atualmente bem difundidos no mercado. Como exemplos destes sistemas, temos:

- LINDO da Lindo Systems (http://www.lindo.com);

- CPLEX da IBM (http://www-01.ibm.com/software/integration/optimization/cplexoptimizer/);

- GPSS da Wolverine Software Corporation (http://www.wolverinesoftware.com);

- ARENA da Paragon Tecnologia Ltda (http://www.paragon.com.br);

- Um outro exemplo é o componente Solver embutido no Excel da Microsoft (http://www.microsoft.com).

É importante salientar que essas ferramentas têm como objetivo a solução de problemas de PO. Dessa forma, o estudante ao utilizá-las não possue nenhuma informação de como se chega à solução. Cada um dos sistemas apresenta peculiaridades de plataformas de utilização e de manuseio e implementam várias técnicas de PO. Todos são sistemas robustos que apresentam maior complexidade para uso e exigem o conhecimento específico de sua utilização, muitas vezes sem exigir o conhecimento das técnicas propriamente ditas.

No ensino de PL são utilizados aplicativos com interfaces baseadas em planilhas eletrônicas (o Solver, por exemplo) e linguagens de modelagem algébricas (o principal exemplo é o LINDO). O uso de planilhas não exige o conhecimento de álgebra, cálculo ou mesmo de uma notação matemática tradicional e o uso de linguagens de modelagem algébricas é familiar aos alunos, que têm bom conhecimento de matemática.

Para definir relacionamentos lógicos de uma solução iterativa ao PPL, através do Método Simplex, são implementados pelos alunos programas computacionais em diferentes linguagens de programação. $\mathrm{O}$ uso deste recurso serve de base para aprimorar o entendimento do processo de solução [Dávalos 2002].

Em [Crisóstomo et al. 2003] os autores apresentam o PL-Edu (Sistema de Programação Linear Educacional), um software que tem no aspecto didático seu principal foco, constituindo-se uma ferramenta que permite o aprendizado da PL através da possibilidade de consulta a um conteúdo didático disponibilizado na forma de um tutorial e, principalmente, pela utilização prática de PL para encontrar soluções ótimas em diversas situações. Contudo, o PL-EDU, não se diferencia muito de um software para solução de PPL's. A única diferença é que o processo de solução é mostrado passo a passo. O usuário, porém, não interage com o sistema, apenas observa o processo de solução.

Em [Molinari 2007] é apresentada a estratégia de ensino e uma amostra de material didático adotado na disciplina de PL. Além das aulas presenciais, duas vezes por semana, os alunos dispõem de um sítio na Internet, que propicia atividades nãopresenciais. No sítio eletrônico, são disponibilizadas as mesmas apresentações computacionais trabalhadas nas aulas presenciais, além de outros materiais didáticos e softwares. As apresentações computacionais possuem uma versão para impressão e uma versão dinâmica que pode ser revista pelo aluno em seu computador em qualquer momento e quantas vezes for necessário. Como na abordagem anterior, o usuário também não interage com o sistema, apenas observa o processo de solução. 


\section{Apresentação da Ferramenta}

A ferramenta E-PL foi construída na Linguagem de Programação JAVA, dado o seu melhor desempenho computacional comparado a outras linguagens de programação. Uma vez escrita em Java, E-PL pode ser facilmente executado ou recompilado em outras plataformas além daquela de origem. Os programas em Java são usualmente compilados para o formato de instruções (bytecodes) de uma plataforma virtual. Assim, uma aplicação Java, no formato bytecode, pode ser executada em qualquer ambiente operacional para $\mathrm{o}$ qual exista uma implementação da Máquina Virtual Java.

Para se adicionar algoritmos e outros recursos ou para a implementação de elementos de interface gráfica, utiliza-se sempre e somente a linguagem Java e sua biblioteca de classes pré-disponíveis. Além disso, essa linguagem contém estruturas de dados completamente apropriadas para a manipulação de vetores e de matrizes numéricas que são a base para os algoritmos de PL.

O E-PL apresenta um menu principal através do qual pode-se consultar o conteúdo didático ou utilizar o ambiente de solução de problemas. O conteúdo didático encontra-se na forma de tutorial baseado em hipertexto de modo a dar mais dinamismo e atratividade às consultas. Este conteúdo apresenta os princípios básicos do método Simplex bem como também abriga conteúdo explicativo sobre a utilização do próprio E-PL.

O ambiente de solução de modelo de PL busca ser o mais intuitivo possível. Para isso foram aplicados os padrões de interfaces para softwares educacionais apresentados em [Reategui 2007]. Além disso, [Flôres e Tarouco 2008] afirmam que a principal característica desejada de um objeto de aprendizagem é a interatividade com o aluno. Dessa forma, a resolução de um modelo no E-PL é guiada pelas ações do aluno. Caso a ação esteja correta o programa continua sua execução, caso contrário, o aluno recebe um aviso que cometeu um engano e uma orientação de como ele deve fazer a escolha. É importante salientar que o software não conserta o engano do aluno. Ele apenas orienta como deve ser o próximo passo para a resolução do problema.

Para solução através do E-PL, deve-se ter o modelo do problema (tarefa que pode ser orientada pelo professor). Após a inserção dos dados, a operação do botão "Calcular" inicia o processo de solução do problema baseado no método Simplex. A Figura 1(a) apresenta a tela inicial do E-PL e a Figura 1(b) apresenta um exemplo de problema a ser solucionado.

Em seguida, os alunos são incentivados a transformar o problema para a forma padrão, pela introdução de variáveis de folga e artificiais, como mostrado na Figura 2(a). O usuário é questionado se há necessidade ou não de algumas dessas variáveis para cada uma das restrições do problema. Caso alguma variável seja inserida erroneamente, o E-PL anuncia o erro ao usuário e apresenta uma justificativa. Em seguida, é montado o quadro do método Simplex, como apresentado na Figura 2(b).

Com o quadro montado, os alunos devem analisá-lo e nele identificar a solução inicial do problema. Os alunos devem identificar a não-otimização da solução obtida (Figura 3(a)) e determinar qual variável sairá e qual variável entrará na base para formar a nova solução, como mostra a Figura 3(b).

Após a seleção da nova base, é montado um novo quadro como apresenta a Figura 


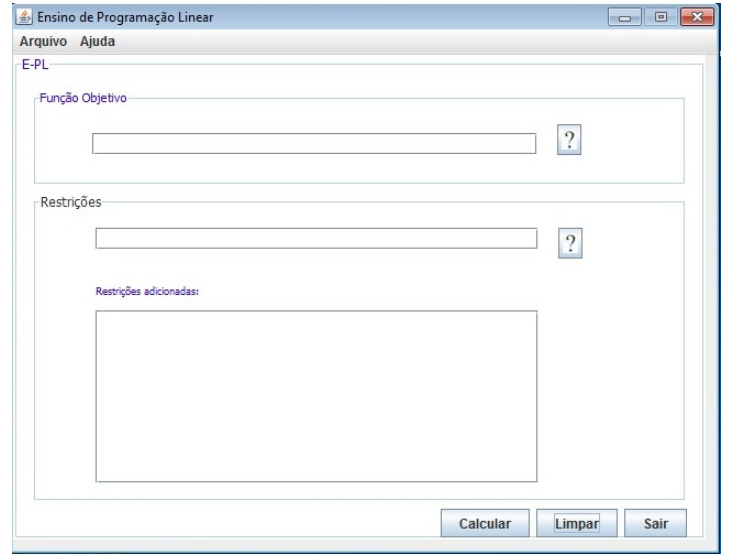

(a) Interface Inicial do E-PL

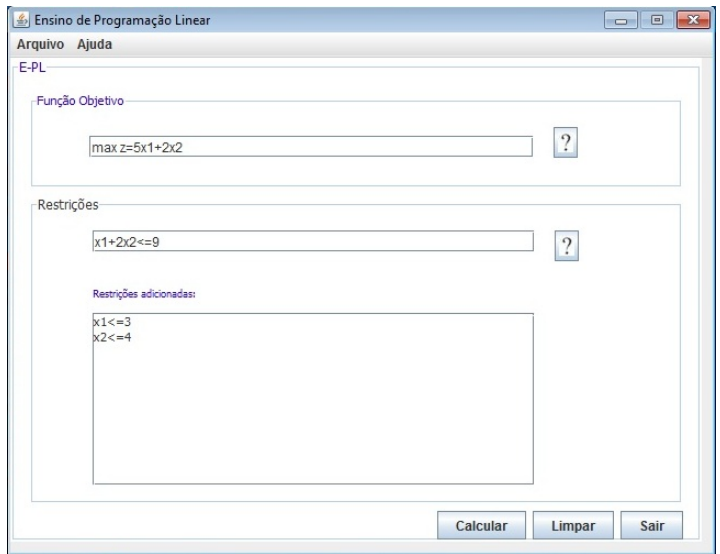

(b) Inserção dos Dados

Figura 1. Início da Solução do PPL

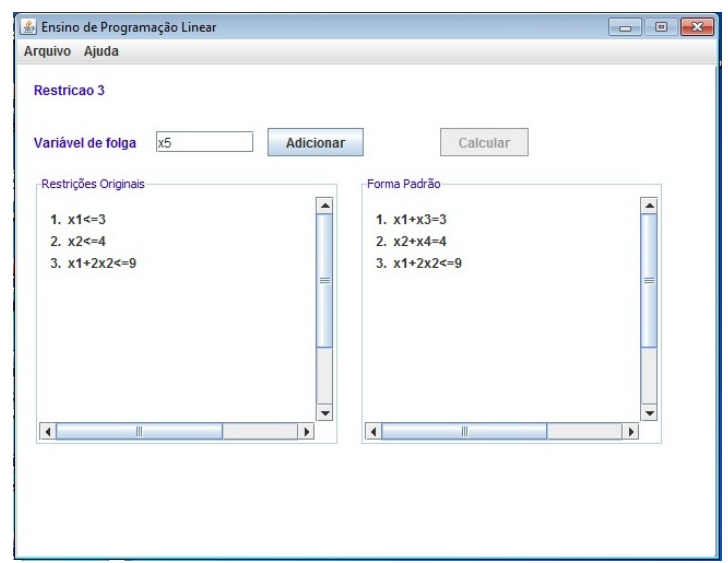

(a) Inserção das Variáveis de Folga

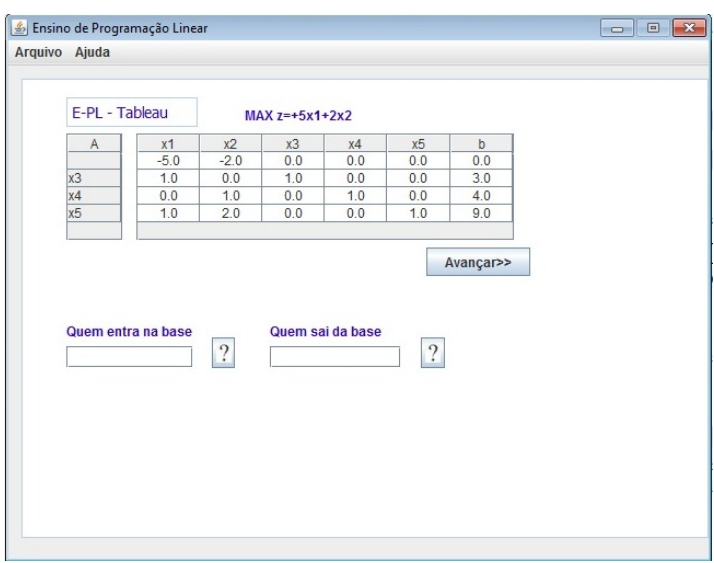

(b) Quadro Inicial

Figura 2. Preparação do Modelo para a Execução do Simplex

4(a). Caso a solução ainda não seja ótima, deve ser feita uma nova determinação das variáveis que entrarão e sairão da base (Figura 4(b) ).

O processo se repete até a obtenção da solução ótima do PPL. A Figura 5(a) mostra que a solução não pode ser melhorada e o quadro final com os valores da Função Objetivo e variáveis é apresentado na Figura 5(b).

Caso o aluno tenha dificuldades em algum passo da resolução ele pode selecionar o botão “?”. Dessa forma, tem-se acesso a uma explicação sobre a ação que ele deve fazer, bem como, uma orientação de como proceder esta ação.

Como processo avaliativo sobre o E-PL, questionários foram disponibilizados de forma on-line para que os alunos com conhecimentos em Programação Linear julgassem alguns quesitos pertinentes, tais como interface, usabilidade, mecanismos de avaliação e princípios pedagógicos, no qual cada um deles continha no mínimo duas perguntas relacionadas aos seus respectivos critérios.

De um espaço amostral de 21 alunos que participaram do processo julgador, geraram-se alguns gráficos à respeito dos formulários e seus quesitos, que são apresenta- 


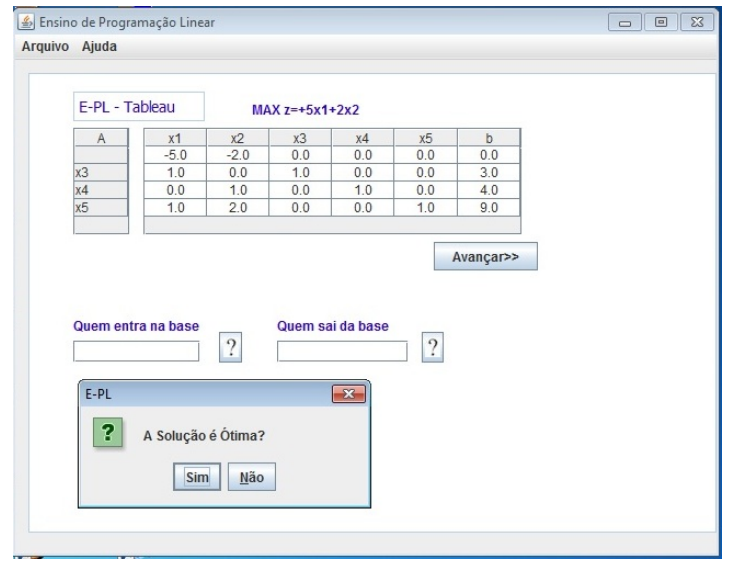

(a) Análise da Otimalidade da Solução

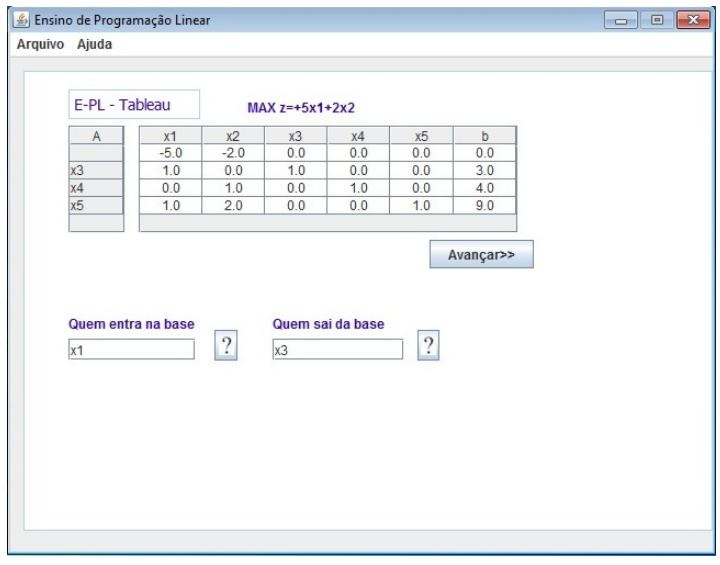

(b) Escolha da Nova Base

Figura 3. Melhoria da Solução

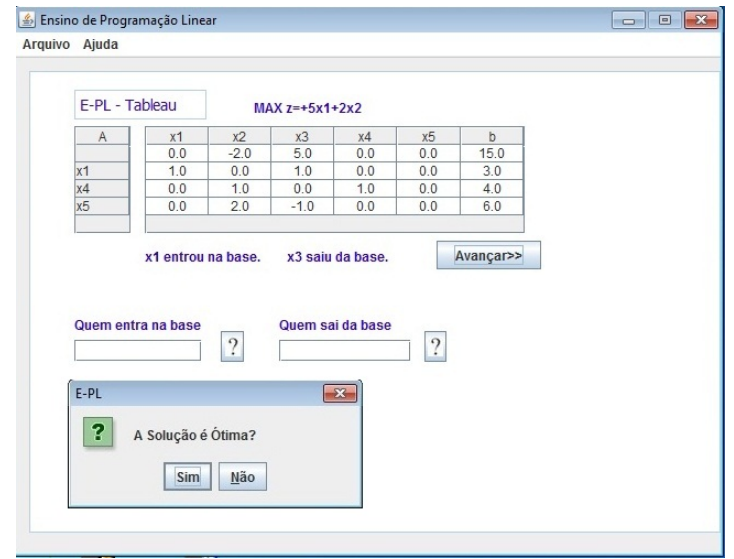

(a) Quadro com a nova Solução

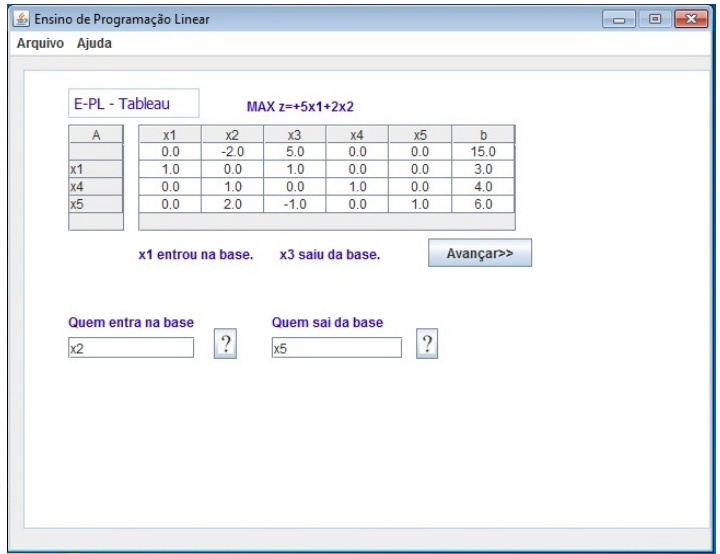

(b) Escolha da Nova Base

Figura 4. Mais um Passo em Busca da Otimalidade

dos estatísticamente nas Figuras 6(a), 6(b), 6(c) e 6(d).

A interface do OA foi bem avaliada de acordo com os alunos (Figura 6(a)), tendo uma boa usabilidade, consequentemente, com o decorrer de sua utilização, conforme ilustra a Figura 6(b), pois sua navegabilidade, adaptação ao usuário, padronização, agrupamento e consistência foram criteriosamente verificados e julgados.

Assim, percebe-se também que a ferramenta obedece ao seu objetivo de criação, pois ajudou na percepção de aprendizagem dos alunos avaliadores, de acordo com a Figura 6(d), confirmando o seu intuito pedagógico com conceito na maioria bom e suficiente.

As perguntas relativas ao quesito de avaliação utilizadas nos questionários, como por exemplo, se existiam tarefas agendadas no ambiente ou atividades estatísticas no E-PL ou testes automáticos, foram de certa forma equilibradas, com uma relevante quantidade acreditando que tais quesitos não se aplicavam ou eram insuficientes para julgar a ferramenta e outra avaliando com os conceitos suficiente e bom em sua maioria, como pode-se verificar na 6(c), fazendo-se perceber que avaliar pode ser algo difícil, já que tende a ser 


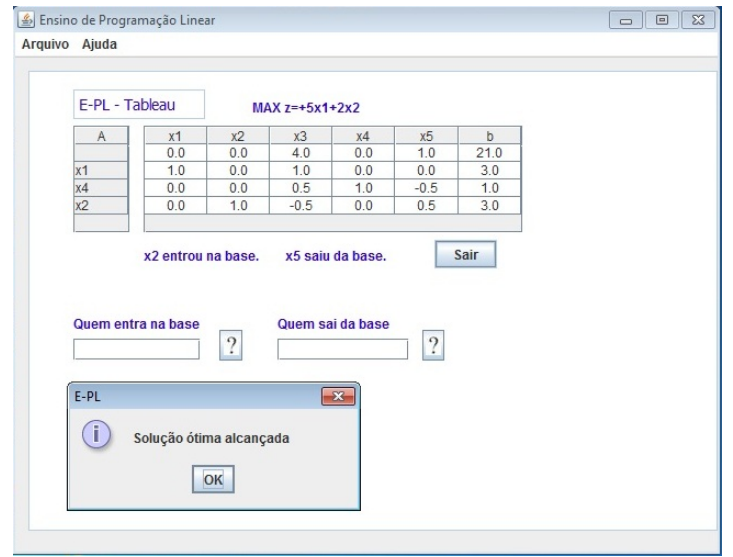

(a) Quadro com a Solução Ótima

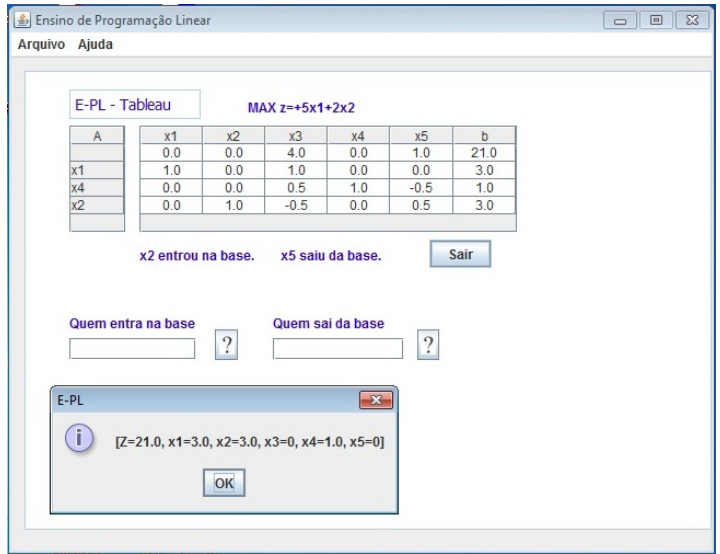

(b) Valores da Função Objetivo e Variáveis

Figura 5. Obtenção da Solução Ótima do PPL

um critério muito subjetivo e dependente da visão de cada usuário.

\section{Conclusões e Trabalhos Futuros}

Para minimizar as dificuldades de ensino-aprendizagem, novas metodologias vêm sendo estudadas, principalmente no que diz respeito ao uso de recursos da tecnologia na educação. Dessa forma, apresenta-se o E-PL como um software cujo principal objetivo é auxiliar aos estudantes no processo de aprendizagem de Programação Linear e oferecer ao docente um novo recurso de ensino desta disciplina, além do desenvolvimento de formas para visualização e análise dos conceitos matemáticos, pois ao se utilizar um sistema que representa graficamente o assunto abordado, o aluno tem no seu perfil uma nova maneira de aprender, assim como a Internet passa a ser para ele uma ferramenta computacional eficiente e instantânea no aprendizado com tais objetos disponíveis.

O OA desenvolvido proporcionou aos estudantes uma maneira diferente de aprender o conteúdo didático, de forma atrativa aos olhos do usuário. A atração do aplicativo vem através das simulações que possibilitam a interação com o aluno, tornando a aula, que utiliza este OA, mais participativa e interessante para o estudante, como demonstraram suas respostas em questionários avaliativos sobre a ferramenta.

A E-PL foi utilizada nas atividades de monitoria e no auxílio ao ensino em Programação Linear oferecido pelo Departamento de Informática ao curso de Bacharelado em Ciência da Computação na Universidade Federal do Piauí, na cidade de Teresina. Seu uso mostrou-se satisfatório, sendo bem aceito pelos alunos.

Durante o período de uso da ferramenta, observou-se um aumento do interesse e da compreensão dos alunos no estudo da disciplina, além da análise crítica através de questionários sobre as interfaces e a usabilidade do E-PL, chegando-se a um conceito mediano bom sobre sua criação e interatividade com o usuário. Portanto, a ferramenta pedagógica desenvolvida apresenta um impacto positivo no aprendizado do referido tema, podendo-se tornar uma maneira de experimentação ativa pelas simulações do assunto abordado. De maneira geral, os alunos consideraram que o E-PL ajudou na aprendizagem do conteúdo, sendo de fácil utilização de acordo com os formulários respondidos sobre sua avaliação. 


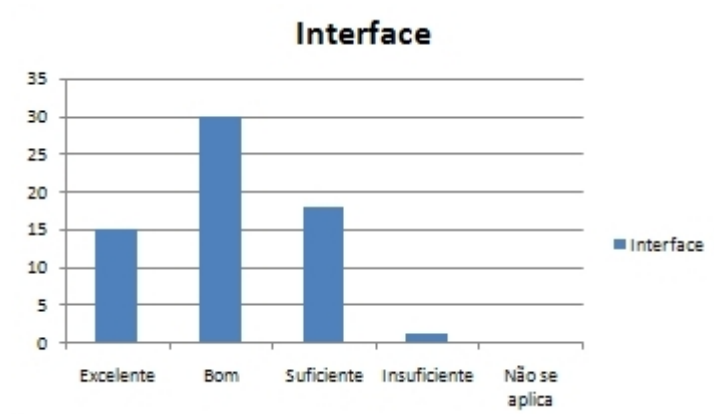

(a) Valores da Avaliação da Interface

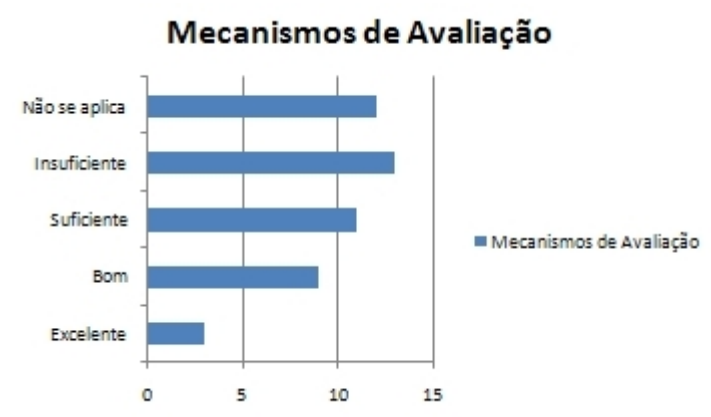

(c) Valores da Avaliação da Interface

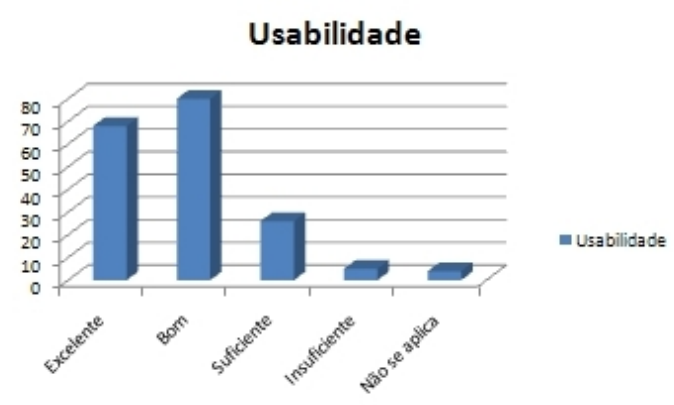

(b) Avaliação da Usabilidade

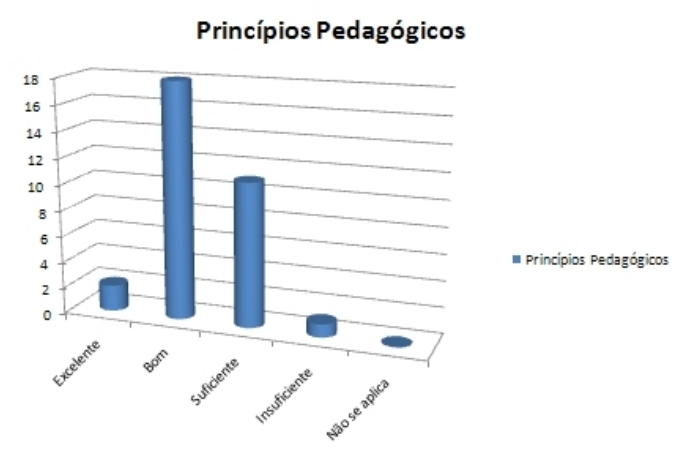

(d) Mecanismos Pedagógicos

Figura 6. Quesitos de Avaliação

Esta versão da ferramenta foi desenvolvida para ser uma aplicação standalone. No entanto, futuramente possuirá recursos web, que possibilitem o acesso remoto, além de permitir troca de ideias, através da interatividade com usuários diversos. Além disso, serão adicionadas mais algumas funcionalidades à ferramenta como a solução gráfica de PPL's e algoritmo Dual-Simplex.

\section{Referências}

Amen, B. e Nunes, L. (2006). Tecnologias de informação e comunicação: contribuições para o processo interdisciplinar no ensino superior. Revista Brasileira de Educação Médica, 30(3):171-180.

Bassani, P. B. S. (2009). Trocas interindividuais no fórum de discussão: um estudo sobre as comunidades de aprendizagem em espaços de educação à distância. In XX Simpósio Brasileiro de Informática na Educação - SBIE.

Bolgheroni, W. e Silveira, I. F. (2008). Software livre aplicado ao ensino de geometria e desenho geométrico. In Anais do XVIII Congresso da SBC - Workshop sobre Informática na Escola (WIE), pp 284-293, Belém-PA. UFPA.

Colin, E. C. (2007). Pesquisa Operacional: 170 Aplicações em Estratégia, Finanças, Logística, Produção, Marketing e Vendas. LTC, 1 edição.

Crisóstomo, V. L., Crisóstomo, D. G., e Freire, F. S. (2003). Aplicação da programação linear em contabilidade de custos e um sistema de otimização educacional. In Congre- 
sos del Instituto Argentino de Profesores Universitarios de Costos (IAPUCO), Punta del Este.

da Silva Brito, G. e Menta, E. (2007). Gepete: Educação bimodal em/para profissionais da educação. In XVIII Simpósio Brasileiro de Informática na Educação - SBIE. Mackenzie.

da Silva Moro, E. L., Estabel, L. B., e Santarosa, L. M. C. (2008). Ambientes virtuais de aprendizagem como vivência educativa, terapêutica e social com adolescentes com fibrose cística internados em isolamento hospitalar. In XIX Simpósio Brasileiro de Informática na Educação - SBIE.

de Almeida Paiva, S. M. (2008). A programação linear no ensino secundário. Dissertação de Mestrado, Universidade Portucalense Infante D. Henrique.

de Souza, P. C., Wazlawick, R. S., e Rosatelli, M. C. (2004). Mediadores sócioconstrutivistas em sistemas de aprendizagem colaborativa. In XV Simpósio Brasileiro de Informática na Educação - SBIE, Manaus/AM. UFAM.

Dávalos, R. V. (2002). Uma abordagem do ensino de pesquisa operacional baseada no uso de recursos computacionais. In XXII Encontro Nacional de Engenharia de Produção, Curitiba.

Flôres, M. L. P. e Tarouco, L. M. R. (2008). Diferentes tipos de objetos para dar suporte a aprendizagem. Novas Tecnologias na Educação, 6(1).

Goldbarg, M. C. e Luna, H. P. L. (2005). Otimização Combinatória e Programação Linear: Modelos e Algoritmos. Campus, 2 edição.

Marquesi, A. L. e Silveira, I. F. (2008). Mapas conceituais aplicados à seleção e organização de objetos de aprendizagem para disciplinas de pré-cálculo em cursos de computação e informática. In Anais do XVIII Congresso da SBC - Workshop sobre Educação em Computação (WEI), pp 137-146, Belém-PA. UFPA.

Molinari, M. (2007). Ensino de programação linear no curso de engenharia de produção civil na utfpr. In Congresso Brasileiro de Educação em Engenharia.

Passerino, L. M., Santarosa, L. M. C., e Tarouco, L. M. R. (2006). Pessoas com autismo em ambientes digitais de aprendizagem: estudo dos processos de interação social e mediação. In XVII Simpósio Brasileiro de Informática na Educação - SBIE, pp 51-60, Brasília/DF. UNB/UCB.

Pereira, L. F. D., Sampaio, F. F., Oliveira, C. E. T., Lapolli, F., e Motta, C. L. R. (2010). Ateliê de objetos de aprendizagem:uma abordagem para o ensino de computação em cursos técnicos. Revista Brasileira de Informática na Educação, 18(3):4-18.

Puccini, A. e Pizzolato, N. (1987). Programação Linear. LTC, Rio de Janeiro.

Reategui, E. (2007). Interfaces para softwares educativos. RENOTE - Revista Novas Tecnologias na Educação, 5(1).

Santos, R. P., Costa, H. A. X., Resende, A. M. P., e Souza, J. M. (2008). O uso de ambientes gráficos para ensino e aprendizagem de estruturas de dados e de algoritmos em grafos. In Anais do XVIII Congresso da SBC - WEI, pp 157-166, Belém/PA. 
Schwarzelmüller, A. F. e Ornellas, B. (2006). Os objetos digitais e suas utilizaçõess no processo de ensino-aprendizagem. In Conferencia Latinoamericana de Objetos de Aprendizaje, Guayaquil/Equador.

Valente, J. A. (1993). Computadores e Conhecimento: repensando a educação, Capítulo Diferentes Usos do Computador na Educação, pp 1-23. Gráfica da UNICAMP, Campinas - SP.

Vieira, F. (2003). Avalição de software educativo: Reflexão para uma análise criteriosa. Disponível em: www.edutec.net/textos/alia/MISC/edmagali2.htm. Acesso em 16 de Agosto de 2011. 